\title{
TBK1 duplication is found in normal tension and not in high tension glaucoma patients of Indian origin
}

\author{
LALIT KAURANI ${ }^{1}$, MANSI VISHAL $^{1,2}$, JHARNA RAY $^{3}$, ABHIJIT SEN $^{4}, \mathrm{KUNAL} \mathrm{RAY}^{2,5 *}$ and \\ ARIJIT MUKHOPADHYAY ${ }^{1 *}$ \\ ${ }^{1}$ Genomics and Molecular Medicine, CSIR-Institute of Genomics and Integrative Biology, New Delhi 110 020, India \\ ${ }^{2}$ Molecular and Human Genetics Division, CSIR-Indian Institute of Chemical Biology, Kolkata 700 032, India \\ ${ }^{3}$ Sachindra Nath Pradhan Centre for Neurosciences, University of Calcutta, Kolkata 700 019, India \\ ${ }^{4}$ Dristi Pradip, Kolkata 700 068, India \\ ${ }^{5}$ Academy of Scientific and Innovative Research (AcSIR), New Delhi 110 025, India
}

\begin{abstract}
[Kaurani L., Vishal M., Ray J., Sen A., Ray K. and Mukhopadhyay A. 2016 TBK1 duplication is found in normal tension and not in high tension glaucoma patients of Indian origin. J. Genet. 95, 459-461]
\end{abstract}

\section{Introduction}

Duplication in TANK-binding kinase 1 (TBK1) gene has been shown to be associated with normal tension glaucoma (NTG), a subtype of the neurodegenerative disease glaucoma, which is the largest cause of irreversible blindness. A specific Taqman assay was used in exploring the genetic contribution of the duplication in TBK1 on 158 NTG and 161 high tension glaucoma (HTG) patients as well as in 197 controls from West Bengal, eastern part of India. We identified two patients in NTG subgroup harbouring the duplication $(1.26 \%)$. Both the patients harboured one extra copy for the genomic segment analysed. No copy number change was observed in the HTG subgroup or in the controls. This is the first report showing presence of duplication in TBK1 in Indian primary open angle glaucoma (POAG) cohort, especially for the NTG subtype. Our finding correlates with the previous findings where duplication in $T B K 1$ is shown to be associated with NTG in various populations.

Glaucoma is a neurodegenerative disease of the eye where vision impairment occurs due to loss of retinal ganglion cells (RGC) and if untreated culminates in irreversible blindness. POAG is the most common subtype affecting $\sim 60$ million people worldwide (Quigley and Broman 2006). The most important risk factor for POAG is higher intraocular pressure (IOP) but almost $40 \%$ of the patients do not represent a high IOP. Thus, POAG patients are usually subdivided into HTG or NTG. Genetic basis of POAG is complex and in addition to Mendelian forms of the disease, single-nucleotide

*For correspondence. E-mail: Arijit Mukhopadhyay, arijit@igib.res.in, arijit@igib.in; Kunal Ray, kunalray@gmail.com, kunalray@acsir.res.in. polymorphism - genomewide association studies (SNP-GWAS) as well as studies involving copy number variations (CNVs) have been reported. Current knowledge on molecular basis of POAG is described in a recent review (Janssen et al. 2013).

Among the POAG cases reported to harbour CNVs, a 780-kb duplication on chromosome 12q14 was found to segregate in a large family of African-American ethnicity for the GLC1P locus with NTG phenotype (Fingert et al. 2011). Subsequently, screening a larger cohort for this region narrowed down the critical duplication interval to $300 \mathrm{~kb}$ which spanned three genes, namely TBK1, XPOT and RASSF3 (Fingert et al. 2011; Kawase et al. 2012). In addition to the African-American population, the duplication was found in NTG patients of Australian, Caucasian and Japanese ethnicities (Kawase et al. 2012; Awadalla et al. 2014; Ritch et al. 2014). To detect the most likely candidate genes influenced by this duplication, a combination of three factors were considered, namely (i) expression in retinal ganglion cells; (ii) altered expression due to the duplication; and (iii) involvement with known glaucoma genes. TBK1 satisfied all the three criteria. Especially, TBK1 with duplication showed increased expression and was found to be a direct interactor of optineurin (OPTN). E50K, the most well known NTG-causing mutation in OPTN was shown to have markedly enhanced binding affinity to TBK1 (Minegishi et al.2013). A recent study identified loss of function mutation in TBK1 gene associated with familial amyotrophic lateral sclerosis and frontotemporal dementia (Freischmidt et al. 2015). It indicates that both gain and loss of function of $\mathrm{T} B K 1$ are detrimental and can lead to disease phenotypes.

Keywords. TBK1 gene; copy number variation; primary open angle glaucoma; normal tension glaucoma. 
In this study, we examined the presence of this specific genomic variant in a glaucoma cohort from West Bengal, India consisting of HTG and NTG subgroups.

\section{Materials and methods}

\section{Selection criteria for study subjects}

The inclusion criteria for patients with glaucoma was (i) optic disc cupping or visual field changes, open angle on gonioscopy and an IOP $>20 \mathrm{~mm}$ of $\mathrm{Hg}$ of glaucoma or (ii) optic disc cupping and visual field changes, open angle on gonioscopy without elevated IOP. Patients of ocular hypertension without any field damage or cupping, and patients with history of ocular trauma were excluded. The samples were recruited after explaining the study to them individually with written consent. This study was approved by the

Table 1. Clinical information for patients with $T B K 1$ duplication.

\begin{tabular}{lcccc}
\hline Patient ID & Gender & Age (years) & IOP (RE) & IOP (LE) \\
\hline GL426 & Male & 54 & 15 & 13 \\
GL582* & Female & 72 & 17.5 & 17.5 \\
\hline
\end{tabular}

IOP, intraocular pressure measure in $\mathrm{mm}$ of $\mathrm{Hg}$. RE, right eye; LE, left eye.

* Patient who also has cataract and hypertension. Both patients were drug-naïve with respect to IOP lowering medication. human ethics committee of the organization and followed the tenets of declaration of Helsinki. Further, we had divided our POAG cohort into NTG and HTG subgroups where NTG selection criteria was IOP $<20 \mathrm{~mm}$ of $\mathrm{Hg}$ in both eyes (untreated) and for HTG, selection criteria was IOP $>23 \mathrm{~mm}$ of $\mathrm{Hg}$ in both eyes (untreated). The final sample set had 158 NTG patients, 161 HTG patients (table 1 in electronic supplementary material at http://www.ias.ac.in/jgenet/) and 197 controls recruited from a large population residing in West Bengal, eastern part of India.

\section{TBK1 duplication quantification by Taqman assay}

For a quantitative and specific assessment of this duplication in our cohort of POAG patients, we used an established TaqMan assay (Fingert et al. 2011). Reactions were performed as per the manufacturer's protocol (Invitrogen, Carlsbad, USA). The specific probes used were: (i) $\mathrm{p} / \mathrm{n}$ 4403326: Taqman copy number reference assay (human RNase P);(ii) $\mathrm{p} / \mathrm{n} 4371355$ : Genotyping master mix; (iii) hs06980763:h: TBK1-specific copy number probe. Fold change was calculated by $2^{\Delta \Delta \mathrm{Ct}}$ based method (Livak and Schmittgen 2001). Quantitative PCR (qPCR) was conducted using 10 ng of DNA; PCR reactions were performed using $5 \mu \mathrm{L}$ of $2 \times$ TaqMan $囚$ Genotyping Master Mix, $0.5 \mu \mathrm{L}$ of TaqMan $\AA$ Copy Number Assay, $20 \times$ working stock and $0.5 \mu \mathrm{L}$ of $20 \times$ TaqMan $\AA$ Copy Number Reference Assay. The qPCR cycling protocol consisted of $95^{\circ} \mathrm{C}$ for $3 \mathrm{~min} ; 40$ cycles of $95^{\circ} \mathrm{C}$ for $5 \mathrm{~s}, 60^{\circ} \mathrm{C}$ for 5 $\mathrm{s}, 72^{\circ} \mathrm{C}$ for $10 \mathrm{~s}$. Experiments were conducted using ROCHE Thermal Cycler LC480 (Indianapolis, USA).

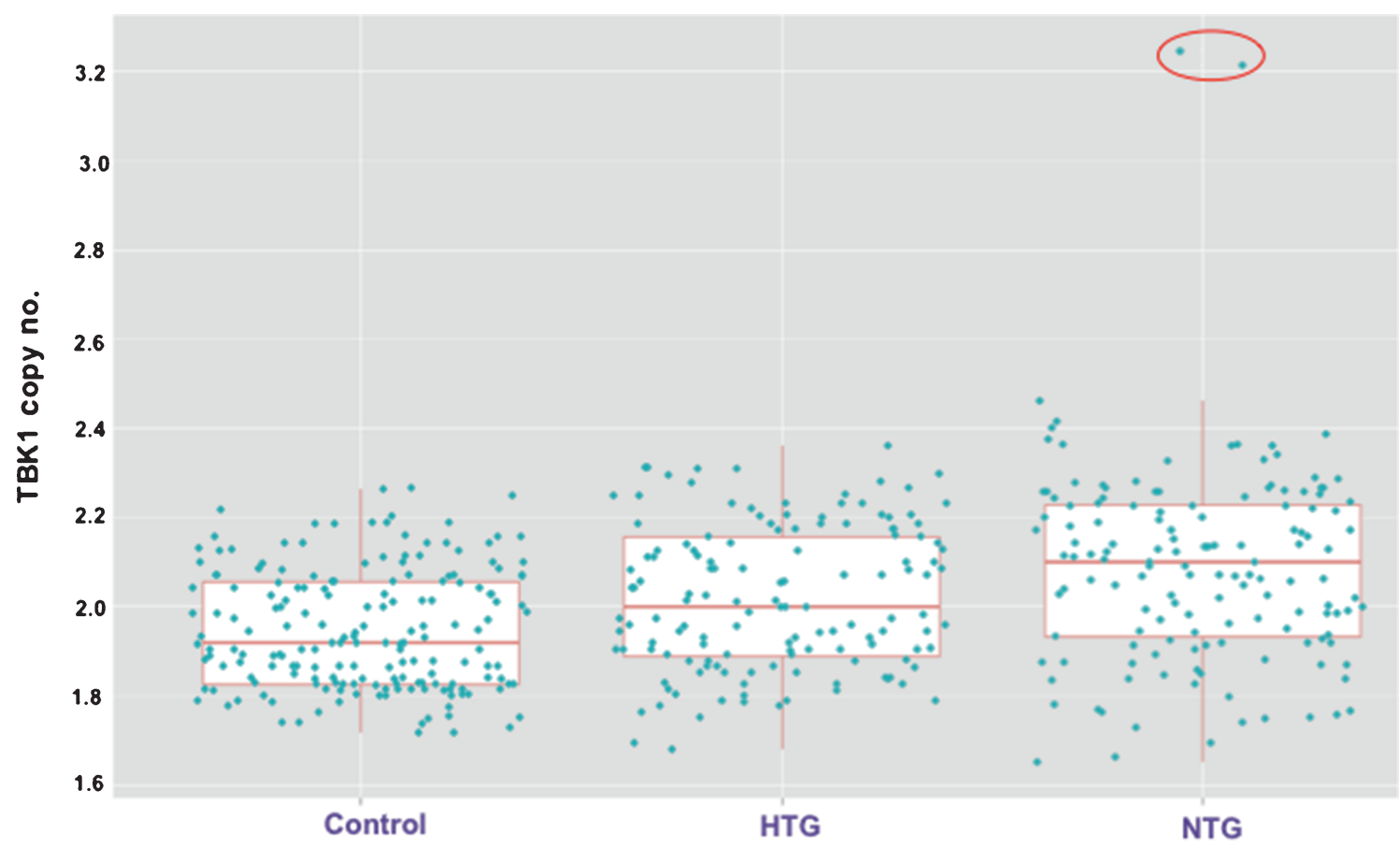

Figure 1. TBK1 duplication in Indian POAG cohort. The figure represents copy number (vertical axis) of TBK1 gene in NTG, HTG and control groups. Each data point represents individual samples. A value of 2.0 indicates normal copy and 3.0 indicate duplication (three copies). The raw data supporting this figure can be found in table 2 in electronic supplementary material. The two NTG samples found to have the duplication are marked by a red ellipse. 


\section{Results and discussion}

We found two NTG patients (table 1) who have the duplication for this region (copy-number change $>1.5$ ) with a significant $P$ value (Grubb's test for outliers, $P<0.01$ ). No other controls or HTG patients showed fold change that indicated duplication (figure 1). The clinical data of HTG and NTG subgroups are provided in table 1 in electronic supplementary material. Raw experimental values and the fold change calculations for all the 465 samples (143 NTG, 140 HTG and 182 controls) that passed the experimental quality criteria are provided in table 2 in electronic supplementary material. Our study supports the fact that the TBK1 duplication is indeed absent in HTG patients thus confirming its specificity for the NTG subgroup.

TBK1 gene encodes a kinase that interacts with the TANK protein (IKK subfamily member), involved in NF $\kappa$ B signalling pathway. It has been reported that in response to TNF $\alpha$, OPTN interacts with TBK1 and recruits it to K63pUb-RIP (receptor interacting protein) complex which activates $\mathrm{NF} \kappa \mathrm{B}$ signalling (Morton et al. 2008). Previous study on glaucomatous astrocytes has shown increased expression of $\mathrm{NF} \kappa \mathrm{B}$ signalling and $\mathrm{TNF} \alpha$ (Nikolskaya et al. 2009). $\mathrm{TNF} \alpha$ activates $\mathrm{NF} \kappa \mathrm{B}$ signalling and has been reported that upregulation of TNF $\alpha$ causes damage to optic nerve head in the POAG patients (Morton et al. 2008). Previous studies have reported that $\mathrm{NF} \kappa \mathrm{B}$ play a key role in autophagy regulation and duplication in $T B K 1$ gene causes increased $\mathrm{NF} \kappa \mathrm{B}$ signalling which contributes the induction of autophagy and thus connecting TBK1 to the biology of glaucomatous neurodegeneration (Trocoli and Djavaheri-Mergny 2011). Our previous work on genome-wide copy number variation for large CNVs in POAG did not detect any CNV in this region due to absence of probes in the microarray platform (Kaurani et al. 2014). It is noteworthy that this duplication is also absent in the database of genomic variants (http://dgv.tcag. $\mathrm{ca} / \mathrm{dgv} / \mathrm{app} / \mathrm{home}$ ) from global population, thus highlighting its detrimental effect. Interestingly, in all the different populations in which this duplication is reported so far is always found to be specific to the NTG subgroup and the duplication frequencies are also consistent (1-2\%). In the field of glaucoma genetics, myocilin (MYOC) is the gene that is most frequently mutated in every population studied so far including India. The frequency of MYOC mutations is $3-4 \%$ across the globe when checked in cohorts of sporadic patients of glaucoma. Thus, although the TBK1 duplication has a low frequency, given the complexity of the genetic aetiology of glaucoma, this seems to be a very important genetic defect that is so far found in each population studied with a consistent frequency and very high specificity only for the NTG subgroup.
Acknowledgements

We acknowledge Dr John Fingert for providing us the DNA sample to be used as positive control to standardize the assay for the duplication. Funding from the Council of Scientific and Industrial Research (CSIR) supported the work (grant numbers BSC-0123 and MLP-0016). LK and MV acknowledge CSIR and Indian Council of Medical Research (ICMR) for their senior research fellowships.

\section{References}

Awadalla M. S., Fingert J. H., Roos B. E., Chen S., Holmes R., Graham S. L. et al. 2014 Copy number variations of TBK1 in Australian patients with primary open angle glaucoma. Am. J. Ophthalmol. 159, 124-130.

Fingert J. H., Robin A. L., Stone J. L., Roos B. R., Davis L. K., Scheetz T. E. et al. 2011 Copy number variations on chromosome $12 \mathrm{q} 14$ in patients with normal tension glaucoma. Hum. Mol. Genet. 20, 2482-2494.

Freischmidt A., Wieland T., Richter B., Ruf W., Schaeffer V., Muller K. et al. 2015 Haploinsufficiency of TBK1 causes familial ALS and fronto-temporal dementia. Nat. Neurosci. 5, 631-636.

Janssen S. F., Gorgels T. G., Ramdas W. D., Klaver C. C., van Duijn C. M., Jansonius N. M. et al. 2013 The vast complexity of primary open angle glaucoma: disease genes, risks, molecular mechanisms and pathobiology. Prog. Retin. Eye Res. 37, 31-67.

Kaurani L., Vishal M., Kumar D., Sharma A., Mehani B., Sharma C. et al. 2014 Gene rich large deletions are overrepresented in POAG patients of Indian and Caucasian origins. Invest. Ophthalmol. Vis. Sci. 24, 3258-3264.

Kawase K., Allingham R. R., Meguro A., Mizuki N., Roos B., Solivan-Timpe F. M. et al. 2012 Confirmation of TBK1 duplication in normal tension glaucoma. Exp. Eye Res. 96, 178180.

Livak K. J. and Schmittgen T. D. 2001 Analysis of relative gene expression data using real-time quantitative PCR and the 2(-Delta Delta C(T)) method. Methods 25, 402-408.

Minegishi Y., Iejima D., Kobayashi H., Chi Z. L., Kawase K., Yamamoto T. et al. 2013 Enhanced optineurin E50K-TBK1 interaction evokes protein insolubility and initiates familial primary open-angle glaucoma. Hum. Mol. Genet. 22, 35593567.

Morton S., Hesson L., Peggie M. and Cohen P. 2008 Enhanced binding of TBK1 by an optineurin mutant that causes a familial form of primary open angle glaucoma. FEBS Lett. 582, 997-1002.

Nikolskaya T., Nikolsky Y., Serebryiskaya T., Zvereva S., Sviridov E., Dezso Z. et al. 2009 Network analysis of human glaucomatous optic nerve head astrocytes. BMC Med. Genomics 2, 24.

Quigley H. A. and Broman A. T. 2006 The number of people with glaucoma worldwide in 2010 and 2020. Br. J. Ophthalmol. 90, 262-267.

Ritch R., Darbro B., Menon G., Khanna C. L., Solivan-Timpe F., Roos B. R. et al. 2014 TBK1 Gene duplication and normaltension glaucoma. JAMA Ophthalmol. 132, 544-548.

Trocoli A. and Djavaheri-Mergny M. 2011 The complex interplay between autophagy and NF-kappaB signaling pathways in cancer cells. Am. J. Cancer Res. 1, 629-649. 